\title{
Identifiability at the boundary for first-order terms
}

\author{
Russell M. Brown *† \\ Department of Mathematics \\ University of Kentucky \\ Lexington, Kentucky 40506-0027 \\ USA \\ Mikko Salo $\ddagger$ \\ Department of Mathematics and Statistics/RNI \\ University of Helsinki \\ P.O. Box 68 \\ 00014 University of Helsinki \\ Finland
}

\begin{abstract}
.
Let $\Omega$ be a domain in $\mathbf{R}^{n}$ whose boundary is $C^{1}$ if $n \geq 3$ or $C^{1, \beta}$ if $n=2$. We consider a magnetic Schrödinger operator $L_{W, q}$ in $\Omega$ and show how to recover the boundary values of the tangential component of the vector potential $W$ from the Dirichlet to Neumann map for $L_{W, q}$. We also consider a steady state heat equation with convection term $\Delta+2 W \cdot \nabla$ and recover the boundary values of the convection term $W$ from the Dirichlet to Neumann map. Our method is constructive and gives a stability result at the boundary.
\end{abstract}

\section{Introduction}

In this note, we consider inverse boundary value problems for the magnetic Schrödinger operator and the Laplacian with a convection term. We recover the boundary values

\footnotetext{
${ }^{*}$ Research supported, in part, by the U.S. National Science Foundation.

${ }^{\dagger}$ Part of this research was carried out while the authors were visiting the University of Washington.

${ }^{\ddagger}$ Research supported, in part, by the Academy of Finland and by the Finnish Academy of Science and Letters, Vilho, Yrjö and Kalle Väisälä Foundation.
} 
of the coefficients from the Dirichlet to Neumann map. Our method is constructive and gives a continuous dependence result. Our method is local in the sense that we only need to know the action of the Dirichlet to Neumann map on functions supported in an arbitrarily small neighborhood of $x_{0}$ in order to recover the value of a coefficient at $x_{0}$. In three dimensions, we work in domains which are $C^{1}$ and require no smoothness on the coefficients beyond continuity. In two dimensions, we need to assume that the boundary is $C^{1, \beta}$ for some $\beta>0$ to push our method through.

The identifiability at the boundary is often a first step in recovering the coefficients in the interior. We expect that we will be able to use the results of this paper to establish interior identifiability with less regularity for the coefficient of the operator and the boundary of the domain.

Before giving more of the background, we give a careful description of the operators we will study. Throughout this note, $\Omega$ will denote a $C^{1}$-domain in $\mathbf{R}^{n}, n \geq 3$ or if $n=2$, a domain which is $C^{1, \beta}$ for some $\beta>0$. Recall that a $C^{1}$-domain (or $C^{1, \beta}$ domain) $\Omega$ is a bounded connected open set and has a $C^{1}$ (or $C^{1, \beta}$ ) defining function $\rho: \mathbf{R}^{n} \rightarrow \mathbf{R}$ with $\Omega=\{x: \rho(x)>0\}, \partial \Omega=\{x: \rho(x)=0\}$. In addition, $\nabla \rho$ does not vanish on $\partial \Omega$. Thus, if we fix $x_{0} \in \partial \Omega$, we may normalize $\rho$ so that $\nabla \rho\left(x_{0}\right)=-\nu\left(x_{0}\right)$ where $\nu(x)$ is the normal to $\partial \Omega$ which points out of $\Omega$.

We will use $L_{W, q}$ to denote a magnetic Schrödinger operator with vector potential $W$ and electric potential $q$. Thus,

$$
L_{W, q}=\sum_{j=1}^{n}\left(\frac{1}{i} \frac{\partial}{\partial x_{j}}+W_{j}\right)^{2}+q .
$$

The vector potential $W: \bar{\Omega} \rightarrow \mathbf{C}^{n}$ is a continuous function on $\bar{\Omega}$ and the electric potential $q$ is in $L^{\infty}(\Omega)$. As we shall see, the electric potential does not enter into our arguments in any essential way. Our argument allows vector potentials that are complex, though the main interest is in real-valued potentials.

We will also consider the Laplacian with a convection term

$$
L_{W}=\Delta+2 W \cdot \nabla
$$

where $\Delta$ is the Laplacian and the convection coefficient $W: \bar{\Omega} \rightarrow \mathbf{C}^{n}$ is a continuous function.

Next, we define the Dirichlet to Neumann operator associated to the operators $L_{W}$ and $L_{W, q}$. We begin with the magnetic Schrödinger operator, $L_{W, q}$. For $f$ in $H^{1 / 2}(\partial \Omega)$, we let $u$ denote the solution of the Dirichlet problem,

$$
\begin{cases}L_{W, q} u=0, & \text { in } \Omega \\ u=f, & \text { on } \partial \Omega .\end{cases}
$$

Our method requires that we be able to solve the Dirichlet problem and thus we assume that zero is not an eigenvalue for the operator $L_{W, q}$ with Dirichlet boundary 
conditions. We define the Dirichlet to Neumann operator acting on $f$ by

$$
\Lambda_{W, q} f=\frac{\partial u}{\partial \nu}+i W \cdot \nu u
$$

In general, $\Lambda_{W, q} f$ is not a function. We give a more careful (and useful) definition of $\Lambda_{W, q} f$ as an element of $H^{-1 / 2}(\partial \Omega)$. If $g$ is in $H^{1}(\Omega)$ (and hence the trace of $g$ on $\partial \Omega$ lies $H^{1 / 2}(\partial \Omega)$-we will use $g$ to denote both the function in $\Omega$ and its trace on the boundary), we have

$$
\begin{gathered}
\left\langle\Lambda_{W, q} f, g\right\rangle=\int_{\Omega} \nabla u(x) \cdot \nabla \bar{g}(x)+W(x) \cdot(i u(x) \nabla \bar{g}(x)-i \bar{g}(x) \nabla u(x)) \\
+(W(x) \cdot W(x)+q(x)) u(x) \bar{g}(x) d x .
\end{gathered}
$$

Since $u$ is a solution of the Dirichlet problem, (1.1), the right-hand side of this expression depends only on the trace of $g$ on $\partial \Omega$ and not on the extension of $g$ into $\Omega$.

In a similar manner, we define the Dirichlet to Neumann operator for the convection operator $L_{W}$. For $f$ in the Sobolev space $H^{1 / 2}(\partial \Omega)$, we let $u$ be the solution of the Dirichlet problem for $L_{W}$,

$$
\begin{cases}L_{W} u=0 & \text { in } \Omega \\ u=f & \text { on } \partial \Omega .\end{cases}
$$

We define $\Lambda_{W} f=\frac{\partial u}{\partial \nu}$ where the precise definition of the normal derivative as an element of $H^{-1 / 2}(\partial \Omega)$ is given by

$$
\left\langle\Lambda_{W} f, g\right\rangle=\int_{\Omega} \nabla u(x) \cdot \nabla \bar{g}(x)-2 \bar{g}(x) W(x) \cdot \nabla u(x) d x .
$$

Again, because $u$ is a solution of (1.3), the right-hand side of (1.4) depends only on the boundary values of $g$ and not the particular extension of $g$ into the interior.

In the theorem below and throughout this paper, we will let $W_{\tan }=W-\nu W \cdot \nu$ denote the tangential component of the boundary values of $W$. With these definitions, we now may state our main results. Our first theorem allows us to recover the tangential component of the boundary values of $W$.

Theorem 1.1 Let $\Omega$ be a $C^{1}$-domain if $n \geq 3$ or $C^{1, \beta}$-domain for some $\beta>0$ if $n=2$. Let $W: \bar{\Omega} \rightarrow \mathbf{C}^{n}$ be a continuous vector potential and $q \in L^{\infty}(\Omega)$, a scalar potential. Assume that zero is not an eigenvalue for $L_{W, q}$. For each $x_{0} \in \partial \Omega$ and $\alpha$, a unit tangent vector to $\partial \Omega$ at $x_{0}$, we may find a family of functions $f_{M}$ with $f_{M}$ supported in a ball of radius $1 / M$ about $x_{0}$,

$$
\lim _{M \rightarrow \infty}\left\|f_{M}\right\|_{L^{2}(\partial \Omega)}=1
$$

and so that

$$
\lim _{M \rightarrow \infty}\left\langle\left(\Lambda_{W, q}-\Lambda_{0,0}\right) f_{M}, f_{M}\right\rangle=\alpha \cdot W\left(x_{0}\right) .
$$

Thus, we may recover the tangential component of $W$ at the boundary from the Dirichlet to Neumann map. 
From this theorem, we easily obtain a continuous dependence result. We also will use $\|T\|$ to denote the operator norm of $T$ on $L^{2}(\partial \Omega)$.

Corollary 1.2 Let $\Omega, W_{j}$ and $q_{j}$ be as in Theorem 1.1. Then we have

$$
\left\|\left(W_{1}-W_{2}\right)_{\tan }\right\|_{L^{\infty}(\partial \Omega)} \leq \sqrt{2}\left\|\Lambda_{W_{1}, q_{1}}-\Lambda_{W_{2}, q_{2}}\right\|
$$

Remark. One deficiency of our results, is that we do not have a sharp criterion which tells us when the operator norm on the right is finite. If $\Omega$ and $W$ are smooth, this norm will be finite.

We have a similar pair of results for the convection operator. As we shall see, the functions $f_{M}$ used in finding the boundary values of the vector potential may also be used to reconstruct the boundary values of the drift term in the convection operator.

Theorem 1.3 Let $\Omega$ be a $C^{1}$-domain if $n \geq 3$ or $C^{1, \beta}$-domain for some $\beta>0$ if $n=2$ and $W: \bar{\Omega} \rightarrow \mathbf{C}^{n}$ a continuous vector-valued function. Assume that 0 is not an eigenvalue for $L_{W}$ in $\Omega$. Then for each $x_{0} \in \partial \Omega$ and $\alpha$ which is a unit tangent vector to $\partial \Omega$ at $x_{0}$, we may find a family of functions $f_{M}$ with $f_{M}$ supported in a ball about $x_{0}$ of radius $1 / M$,

$$
\lim _{M \rightarrow \infty}\left\|f_{M}\right\|_{L^{2}(\partial \Omega)}=1
$$

and

$$
\lim _{M \rightarrow \infty}\left\langle\left(\Lambda_{W}-\Lambda_{0}\right) f_{M}, f_{M}\right\rangle=-\left(i \alpha+\nu\left(x_{0}\right)\right) \cdot W\left(x_{0}\right) .
$$

As a consequence, we may recover the boundary values of $W$.

Remark. If $W$ is real-valued, then the maximum principle implies that 0 is not an eigenvalue for $L_{W}$, thus our hypothesis is satisfied in the case that is most interesting.

Corollary 1.4 If $\Omega, W_{1}$ and $W_{2}$ as in the theorem, then we have

$$
\left\|W_{1}-W_{2}\right\|_{L^{\infty}(\partial \Omega)} \leq \sqrt{3}\left\|\Lambda_{W_{1}}-\Lambda_{W_{2}}\right\|
$$

Ours are not the first results on identifiability at the boundary for first-order terms. Nakamura, Sun and Uhlmann [9] consider the magnetic Schrödinger operator in smooth domains in $\mathbf{R}^{n}, n \geq 3$, and with $C^{\infty}$-coefficients. Their methods should also give boundary identifiability when $n=2$. They go on to study the problem of recovery in the interior. The problem of identifiability of a vector potential was first studied by Sun [11]. Other authors who have considered this problem include Eskin and Ralston [5] and Tolmasky [13]. In his Ph.D. thesis [10], Salo gives recovery of a continuous vector potential in domains that are $C^{1,1}$. We relax the regularity hypothesis for the boundary determination to just $C^{1}$.

Interior identifiability of a convection term has been considered by several authors including Cheng, Nakamura and Somersalo [3] and Salo [10] in dimensions three and 
higher and Cheng and Yamamoto [4], Tong, Cheng and Yamamoto [14] and Tamasan [12] in two dimensions. Tong, Cheng, and Yamamoto work with $L^{p}$ coefficients and do not need identifiability at the boundary as a first step towards interior identifiability. Cheng, Nakamura and Somersalo prove a result on identifiability at the boundary in dimensions three and higher. Their methods likely extend to two dimensions. Isakov [7] proves a theorem on identifiability at the boundary for nonlinear equations that includes the operators we consider, however he requires additional smoothness assumptions on the domain and the coefficients. Salo [10] proves the coefficient $W$ is uniquely determined by the Dirichlet to Neumann map in dimensions three and higher. Salo's argument at the boundary uses the singular solutions of Alessandrini [1]. It would be interesting to adapt Alessandrini's techniques to give a reconstruction of $W$ at the boundary, rather than just showing uniqueness.

Our proof for recovering $W$ at the boundary will follow the argument used by Salo for recovering the the vector potential for a magnetic Schrödinger operator [10]. Salo's method is based on work of Brown [2]. Our proof requires estimates for the $L^{2}(\Omega)$ norm of one of the functions appearing in the expressions (1.2) and (1.4) defining the Dirichlet to Neumann maps. By contrast, the recovery of a conductivity in the work of Brown [2] only requires the standard energy estimate for the gradient of a solution. The new estimate is obtained using methods developed by Jerison and Kenig [8] in their study of harmonic functions in Lipschitz domains.

In our proofs, we will use $c$ and $C$ to denote constants that depend only on the domain $\Omega$.

\section{Construction of approximate solutions}

In this section, we write down the boundary functions $f_{M}$ that we will use to recover $W$. We will present these functions as the boundary values of an approximate solution to the Laplacian. The remainder of this section is devoted to proving estimates for solutions of boundary value problems with this data.

We will fix a point $x \in \partial \Omega$ and a unit vector $\alpha$ which is tangent to $\partial \Omega$. By a change of coordinates we may assume that $x$ is the origin and that the normal to $\partial \Omega$ at the origin is $-e_{n}$. We recall that $\rho$ is a defining function for $\Omega$ and we normalize $\rho$ so that $|\nabla \rho(0)|=1$.

Next, we let $\omega$ be a modulus of continuity for $\nabla \rho$. Thus $\omega:[0, \infty) \rightarrow[0, \infty)$ is a strictly increasing continuous function with $\omega(0)=0$. We let $\eta \in C_{0}^{\infty}\left(\mathbf{R}^{n}\right)$ be a function which is supported in the ball of radius $1 / 2$ centered at 0 and which satisfies

$$
\int_{\mathbf{R}^{n-1}} \eta\left(x^{\prime}, 0\right)^{2} d x^{\prime}=1
$$

We put $\eta_{M}(x)=\eta\left(M\left(x^{\prime}, \rho(x)\right)\right.$ where we are using $x^{\prime}=\left(x_{1}, \ldots, x_{n-1}\right)$ to denote the first $n-1$ coordinates. For $M$ large, we will have $\eta_{M}$ supported in a ball of radius 
$1 / M$. Finally, we define $u_{0}=v_{0}$ by

$$
u_{0}(x)=v_{0}(x)=\eta_{M}(x) e^{N(i \alpha \cdot x-\rho(x))} .
$$

Since our defining function for $\Omega, \rho$, is $C^{1}$, we have that $u_{0}$ and $v_{0}$ are compactly supported and $C^{1}$. The parameters $N$ and $M$ are related by the equation

$$
M^{-1} \omega\left(M^{-1}\right)=N^{-1}
$$

Note that since $\omega$ is increasing on $[0, \infty)$, the equation (2.3) will have exactly one solution $N$ for each $M$. Also, since $\lim _{t \rightarrow 0^{+}} \omega(t)=0$, there is a value $M_{0}$ so that $\omega\left(M^{-1}\right)<1$ if $M>M_{0}$. We will assume that $M>M_{0}$ and then we have $M<N$.

Our next Lemma gives several technical facts that will be needed in the estimates that follow. In this lemma and below, we use $\delta(x)$ to denote the distance from $x$ to the boundary of $\Omega$.

Lemma 2.1 Let $\eta$ be continuous and supported in $B_{1}(0)$. Set $\eta_{M}(x)=\eta\left(M\left(x^{\prime}, \rho(x)\right)\right)$. Let $N$ and $M$ be related as in (2.3). We have the following

$$
\lim _{M \rightarrow \infty} M^{n-1} N \int_{\Omega} \exp (-2 N \rho(x)) \eta_{M}(x) d x=\frac{1}{2} \int_{\mathbf{R}^{n-1}} \eta\left(x^{\prime}, 0\right) d x^{\prime}
$$

and

$$
\left|\int_{\Omega} \delta(x)^{k} \exp (-2 N \rho(x)) \eta_{M}(x) d x\right| \leq C M^{1-n} N^{-k-1} .
$$

The proof of this Lemma is not difficult and we omit the details. We will also need Hardy's inequality. If $f$ is a function in $H_{0}^{1}(\Omega)$, then Hardy's inequality states that

$$
\int_{\Omega}\left|\frac{f(x)}{\delta(x)}\right|^{2} d x \leq C \int_{\Omega}|\nabla f(x)|^{2} d x .
$$

This estimate holds if the domain $\Omega$ is Lipschitz and the constant depends only on the geometry of $\Omega$.

Lemma 2.2 Let $u_{0}$ be as defined in (2.2). For $M>M_{0}$, we have

$$
\left\|\Delta u_{0}\right\|_{H^{-1}(\Omega)} \leq C \omega\left(M^{-1}\right) M^{(1-n) / 2} N^{1 / 2} .
$$

Proof. We compute

$$
\begin{aligned}
\Delta u_{0}(x)=\operatorname{div}( & \left.\nabla \eta_{M}(x) \exp (N(i \alpha \cdot x-\rho(x)))\right) \\
& +N \operatorname{div}\left(\eta_{M}(x)\left(e_{n}-\nabla \rho(x)\right) \exp (N(i \alpha \cdot x-\rho(x)))\right) \\
& +N \nabla \eta_{M}(x) \cdot\left(i \alpha-e_{n}\right) \exp (N(i \alpha \cdot x-\rho(x))) \\
& +N^{2} \eta_{M}(x)\left(i \alpha-e_{n}\right) \cdot(i \alpha-\nabla \rho(x)) \exp (N(i \alpha \cdot x-\rho(x))) \\
=\quad F_{1}(x) & +F_{2}(x)+F_{3}(x)+F_{4}(x) .
\end{aligned}
$$


We estimate the contribution from each term. To study $F_{1}(x)$, we pair with a test function $f$ in $H_{0}^{1}(\Omega)$, use Cauchy-Schwarz and then Lemma 2.1 to obtain

$$
\begin{aligned}
\left|\left\langle f, F_{1}\right\rangle\right| & =\left|\int_{\Omega} \nabla \eta_{M}(x) \cdot \nabla f(x) \exp (-N(i \alpha \cdot x+\rho(x))) d x\right| \\
& \leq C\|\nabla f\|_{L^{2}(\Omega)} M^{(3-n) / 2} N^{-1 / 2} .
\end{aligned}
$$

Recalling the relation between $M$ and $N$, (2.3), we obtain

$$
\left\|F_{1}\right\|_{H^{-1}(\Omega)} \leq C \omega\left(M^{-1}\right) M^{(1-n) / 2} N^{1 / 2} .
$$

To estimate $F_{2}$, we use Cauchy-Schwarz, Lemma 2.1, and our assumption that $\left|\nabla \rho(x)-e_{n}\right| \leq \omega\left(M^{-1}\right)$ on the support of $\eta_{M}$ to obtain that

$$
\begin{aligned}
\left|\left\langle f, F_{2}\right\rangle\right| & \leq N \omega\left(M^{-1}\right)\|\nabla f\|_{L^{2}(\Omega)}\left(\int_{\Omega} \eta_{M}(x)^{2} \exp (-2 N \rho(x)) d x\right)^{1 / 2} \\
& \leq C \omega\left(M^{-1}\right) M^{(1-n) / 2} N^{1 / 2}
\end{aligned}
$$

To estimate $F_{3}$, we use Cauchy-Schwarz, Hardy's inequality (2.4) and Lemma 2.1 to obtain

$$
\begin{aligned}
\left\langle f, F_{3}\right\rangle & \leq C N\left(\int_{\Omega}\left(\frac{f(x)}{\delta(x)}\right)^{2} d x\right)^{1 / 2}\left(\int_{\Omega} \delta(x)^{2}\left|\nabla \eta_{M}(x)\right|^{2} \exp (-2 N \rho(x)) d x\right)^{1 / 2} \\
& \leq C \frac{M}{N} M^{(1-n) / 2} N^{1 / 2}\|\nabla f\|_{L^{2}(\Omega)}
\end{aligned}
$$

Recalling that $\omega\left(M^{-1}\right)=M / N$ (see (2.3)), we obtain the estimate of the Lemma.

For the term $F_{4}$, we use that $\nabla \rho(0)=e_{n}$, is the unit inner normal to $\partial \Omega$, and $\alpha$ is a unit tangent vector to $\Omega$ to obtain $\left(i \alpha-e_{n}\right) \cdot\left(i \alpha-e_{n}\right)=0$. Hence, by the continuity of $\nabla \rho$, it follows that

$$
\left|\left(i \alpha-e_{n}\right) \cdot(i \alpha-\nabla \rho(x))\right| \leq C \omega\left(M^{-1}\right), \quad x \in \operatorname{supp} \eta_{M}
$$

Thus, as with $F_{3}$, we use Cauchy-Schwarz, Lemma 2.1 and Hardy's inequality (2.4) to obtain

$$
\begin{aligned}
\left\langle f, F_{4}\right\rangle & \leq C N^{2}\left(\int_{\Omega}\left(\frac{f(x)}{\delta(x)}\right)^{2} d x\right)^{1 / 2} \omega\left(M^{-1}\right)\left(\int_{\Omega} \delta(x)^{2} \eta_{M}(x)^{2} \exp (-2 N \rho(x)) d x\right)^{1 / 2} \\
& \leq C \omega\left(M^{-1}\right) M^{(1-n) / 2} N^{1 / 2}\|\nabla f\|_{L^{2}(\Omega)} .
\end{aligned}
$$

The desired estimate for $\left\|\Delta u_{0}\right\|_{H^{-1}(\Omega)}$ follows from the inequalities above for $F_{1}$ to $F_{4}$. 
Our next step is to estimate $L_{W, q} u_{0}$ and $L_{W} u_{0}$. These estimates are easy consequences of Lemma 2.2.

Lemma 2.3 Let $\Omega, u_{0}$ be as in Lemma 2.2. Suppose $W$ and $q$ are in $L^{\infty}(\Omega)$. Then we have the estimates

$$
\left\|L_{W, q} u_{0}\right\|_{H^{-1}(\Omega)} \leq C M^{(1-n) / 2} N^{1 / 2} \omega\left(M^{-1}\right)
$$

and

$$
\left\|L_{W} u_{0}\right\|_{H^{-1}(\Omega)} \leq C M^{(1-n) / 2} N^{1 / 2} \omega\left(M^{-1}\right)
$$

Proof. The proof of this lemma amounts to showing that $\left\|L_{W} u_{0}-\Delta u_{0}\right\|_{H^{-1}(\Omega)}$ is smaller than $\left\|\Delta u_{0}\right\|_{H^{-1}(\Omega)}$ as $M \rightarrow \infty$. The additional terms are lower order than those we studied in Lemma 2.2 and may be estimated using the tools introduced in the proof of that Lemma. We omit the details.

Our next step is to construct solutions of the Dirichlet problem for $L_{W, q}$ (or $L_{W}$ ) and the Laplacian. We let $v=v_{0}+v_{1}$ where $v_{1}$ is the solution of

$$
\left\{\begin{array}{l}
\Delta v_{1}=-\Delta v_{0}, \\
v_{1} \in H_{0}^{1}(\Omega)
\end{array}\right.
$$

It is easy to see that $v$ is a solution to the Dirichlet problem for the Laplacian in $\Omega$ with boundary data $u_{0}$.

When discussing the magnetic Schrödinger operator, we will let $u=u_{0}+u_{1}$ where $u_{1}$ is a solution of

$$
\left\{\begin{array}{l}
L_{W, q} u_{1}=-L_{W, q} u_{0}, \quad \text { in } \Omega \\
u_{1} \in H_{0}^{1}(\Omega) .
\end{array}\right.
$$

It is clear that $u$ is a solution of the Dirichlet problem for $L_{W, q}$ with boundary data $u_{0}$.

When discussing the convection equation, we make the analogous definition for $u=u_{0}+u_{1}$ by setting

$$
\left\{\begin{array}{l}
L_{W} u_{1}=-L_{W} u_{0} \quad \text { in } \Omega \\
u_{1} \in H_{0}^{1}(\Omega) .
\end{array}\right.
$$

And we see that $u$ is a solution of the Dirichlet problem for $L_{W}$ with boundary data $u_{0}$.

Our first estimates are the energy estimates for $u_{1}$ and $v_{1}$. These follow immediately from our estimates of Lemma 2.3 and standard Hilbert space existence theory.

Lemma 2.4 Let $u_{1}$ be the solution of (2.6) or (2.7) and let $v_{1}$ be the solution of (2.5). Assume also that 0 is not an eigenvalue for $L_{W, q}\left(\right.$ or $\left.L_{W}\right)$ in $\Omega$. Then we have

$$
\begin{aligned}
\left\|\nabla u_{1}\right\|_{L^{2}(\Omega)} & \leq C \omega\left(M^{-1}\right) N^{1 / 2} M^{(1-n) / 2} \\
\left\|\nabla v_{1}\right\|_{L^{2}(\Omega)} & \leq C \omega\left(M^{-1}\right) N^{1 / 2} M^{(1-n) / 2}
\end{aligned}
$$


Our next step is to estimate the $L^{2}$-norm of $v_{1}$, the solution of (2.5).

Lemma 2.5 Let $n \geq 3$ and $\Omega$ be a $C^{1}$-domain, then the solution of (2.5) satisfies

$$
\left\|v_{1}\right\|_{L^{2}(\Omega)} \leq C M^{(1-n) / 2} N^{-1 / 2} .
$$

Note that away from the support of $v_{0}$, the function $v_{1}$ is harmonic. Thus we expect that $v_{1}$ decays like the Green's function for $\Omega, G(x, y)$. If $n \geq 5$, then the elementary estimate $G(x, y) \leq|x-y|^{2-n}$ is enough to imply that $v_{1}$ is in $L^{2}(\Omega)$. When $n=3$ and 4 , we need additional estimates which rely on the vanishing of $G$ on the boundary of $\Omega$. When $n=2$, we need additional regularity on the boundary to push the argument through.

Proof. We begin the proof by using the Poincaré inequality in a ball centered at 0 and of radius $100 M^{-1}$ to obtain

$$
\begin{aligned}
\int_{\Omega \cap B_{100 M^{-1}}(0)}\left|v_{1}(x)\right|^{2} d x \leq C M^{-2} \int_{\Omega}\left|\nabla v_{1}(x)\right|^{2} d x & =C M^{-1-n} N \omega\left(M^{-1}\right)^{2} \\
& =C M^{1-n} N^{-1}
\end{aligned}
$$

where we have also used Lemma 2.4 and (2.3). We extend $\left|v_{1}\right|$ by setting $\left|v_{1}\right|=0$ outside $\Omega$ and the resulting function will be sub-harmonic in $\mathbf{R}^{n} \backslash B_{M^{-1}}(0)$. Thus, we may use the mean-value inequality for sub-harmonic functions and the $L^{2}$-estimate (2.8) to conclude that

$$
\left|v_{1}(x)\right| \leq C(M / N)^{1 / 2}, \quad \text { if } 2 M^{-1} \leq|x| \leq 4 M^{-1}
$$

We let $w(x)=\omega^{x}\left(\partial \Omega \cap B_{4 M^{-1}}(0)\right)$ where $\omega^{x}$ is the harmonic measure for $\Omega$. We observe that the Hölder continuity of harmonic functions and the Harnack principle imply that $w(x) \geq c$ on $\Omega \cap B_{2 M^{-1}}(0)$ (see [8, Lemma 5.2]). Thus by the maximum principle and (2.9), we conclude

$$
\left|v_{1}(x)\right| \leq C w(x)(M / N)^{1 / 2}, \quad x \in \Omega \backslash B_{2 M^{-1}}(0) .
$$

According to Lemma 5.8 in Jerison and Kenig [8] for $M$ large, we have

$$
w(x) \leq C M^{2-n} G\left(x, A_{4 M^{-1}}(0)\right), \quad x \in \Omega \backslash B_{8 M^{-1}}(0) .
$$

Here, for $x \in \partial \Omega$ and $r$ small, we let $A_{r}(x)$ denote a point in $\Omega$ with $\left|A_{r}(x)-x\right|=r$ and $\delta\left(A_{r}(x)\right) \geq c r$. Also, $G(x, y) \geq 0$ is the Green's function for the Laplacian on $\Omega$. From the easy upper bound for $G$,

$$
G(x, y) \leq C|x-y|^{2-n},
$$

and the Hölder continuity of harmonic functions in $\Omega$ (see [8, Lemma 5.1]), we have

$$
G\left(x, A_{4 M^{-1}(0)}\right) \leq C_{\kappa} M^{-\kappa}|x|^{2-n-\kappa}, \quad x \in \Omega \backslash B_{8 M^{-1}}(0) .
$$


In a Lipschitz domain, the estimate (2.13) holds for some $\kappa$ which tends to zero as the Lipschitz constant increases. Since $\Omega$ is $C^{1}$, the estimate (2.13) will hold for any $\kappa$ in $(0,1)$. This is well-known, but we were not able to locate a reference. To establish the result for $C^{1}$ domains, one may construct barrier functions in a cone by separation of variables.

From $(2.10),(2.11)$ and $(2.13)$, we conclude for any $\kappa$ in $(0,1)$, there is a constant $C_{\kappa}$ so that

$$
\left|v_{1}(x)\right| \leq C_{\kappa}(M / N)^{1 / 2}(M|x|)^{2-n-\kappa}, \quad|x|>8 M^{-1} .
$$

Integrating this inequality, gives

$$
\int_{|x|>8 M^{-1}}\left|v_{1}(x)\right|^{2} d x \leq C M^{1-n} N^{-1}
$$

To obtain this estimate, we must choose $\kappa>0$ when $n=4$ and $\kappa>1 / 2$ if $n=3$. For $n=2$, see the remark after the proof of Theorem 1.3. If $n \geq 5$, we only need the easy upper bound for $G(2.12)$; the Hölder estimate is not needed. Combining the Poincaré inequality (2.8) and our last estimate gives the result of the theorem.

With our main estimates established, we proceed to summarize in a technical Lemma the consequences of Lemma 2.5 and Lemma 2.4 that we will need to complete our proof.

Lemma 2.6 With $u=u_{0}+u_{1}$ the solution of the magnetic Schrödinger equation or convection operator and $v=v_{0}+v_{1}$ the solution of the Laplacian constructed above, we have the following estimates:

$$
\begin{aligned}
\left\|u_{0}\right\|_{L^{2}(\Omega)} & \leq C M^{(1-n) / 2} N^{-1 / 2} \\
\left\|\nabla u_{1}\right\|_{L^{2}(\Omega)} & \leq C M^{(1-n) / 2} N^{1 / 2} \omega\left(M^{-1}\right) \\
\left\|u_{1} / \delta\right\|_{L^{2}(\Omega)} & \leq C M^{(1-n) / 2} N^{1 / 2} \omega\left(M^{-1}\right) \\
\|u\|_{L^{2}(\Omega)} & \leq C M^{(1-n) / 2} N^{1 / 2} \omega\left(M^{-1}\right) \\
\left\|\delta \nabla u_{0}\right\|_{L^{2}(\Omega)} & \leq C M^{(1-n) / 2} N^{-1 / 2} \\
\|v\|_{L^{2}(\Omega)} & \leq C M^{(1-n) / 2} N^{-1 / 2} \\
\|\delta \nabla v\|_{L^{2}(\Omega)} & \leq C M^{(1-n) / 2} N^{-1 / 2} \\
\left\|\nabla v_{1}\right\|_{L^{2}(\Omega)} & \leq C M^{(1-n) / 2} N^{1 / 2} \omega\left(M^{-1}\right) \\
\left\|v_{1} / \delta\right\|_{L^{2}(\Omega)} & \leq C M^{(1-n) / 2} N^{1 / 2} \omega\left(M^{-1}\right)
\end{aligned}
$$

Proof. The estimates (2.14) and (2.18) for $u_{0}$ are easy because we have an explicit formula (2.2) for $u_{0}$. The estimate (2.15) for $\nabla u_{1}$ and the estimate (2.21) for $\nabla v_{1}$ are restatements of the energy estimates in Lemma 2.4. The estimates in (2.16) and (2.22) follow from (2.15) and (2.21) and the Hardy inequality, (2.4). The estimate (2.17) for $u=u_{0}+u_{1}$ follows from the estimate (2.14) for $u_{0}$, the estimate for $\nabla u_{1}$, 
(2.15), and the Poincaré inequality in $\Omega$. The estimate (2.19) follows from (2.14) (recall $u_{0}=v_{0}$ ) and the estimate for $v_{1}$ in Lemma 2.5. Finally, the estimate (2.20) follows from (2.19) by using interior estimates for harmonic functions [6, Theorem 2.10] and the Hardy-Littlewood maximal functions as in Salo [10, Lemma 5.5].

Remark. The estimate (2.17) for the $L^{2}$ norm of the solution to the magnetic Schrödinger operator is probably not sharp. However, it is sufficient for our purposes.

\section{Proofs of the main results.}

We are ready to give the last few steps needed to prove our main theorem.

Proof of Theorem 1.1. We fix $x_{0}$ in the boundary and wish to recover the tangential component of $W$ at $x_{0}$. As above, we assume that $x_{0}=0$ and that $-e_{n}$ is the unit outer normal to $\partial \Omega$ at $x_{0}$. For $M$ sufficiently large, we define

$$
f_{M}(x)=M^{(n-1) / 2} u_{0}(x)
$$

where $u_{0}$ is the function defined in (2.2). Thanks to the normalization of $\eta$ in (2.1) it is easy to see that

$$
\lim _{M \rightarrow \infty} \int_{\partial \Omega}\left|f_{M}(x)\right|^{2} d x=1
$$

which gives the conclusion (1.5) in the statement of the theorem.

Using that the Dirichlet to Neumann map for the Laplacian, $\Lambda_{0,0}$, is self-adjoint, we write

$$
\begin{aligned}
\left\langle\left(\Lambda_{W, q}-\Lambda_{0,0}\right) f_{M}, f_{M}\right\rangle= & M^{n-1} \int_{\Omega} W(x) \cdot(i u(x) \nabla \bar{v}(x)-i \bar{v}(x) \nabla u(x)) \\
& +(W(x) \cdot W(x)+q(x)) u(x) \bar{v}(x) d x \\
= & M^{n-1}\left(i \int_{\Omega} u_{0}(x) W(x) \cdot \nabla \bar{v}_{0}(x)-\bar{v}_{0}(x) W(x) \cdot \nabla u_{0}(x) d x\right. \\
& +i \int_{\Omega} u_{0}(x) W(x) \cdot \nabla \bar{v}_{1}(x) d x \\
& +i \int_{\Omega} u_{1}(x) W(x) \cdot \nabla \bar{v}(x) d x \\
& -i \int_{\Omega} \bar{v}_{1}(x) W(x) \cdot \nabla u_{0}(x) d x \\
& -i \int_{\Omega} \bar{v}(x) W(x) \cdot \nabla u_{1}(x) d x \\
& \left.+\int_{\Omega}(W(x) \cdot W(x)+q(x)) u(x) \bar{v}(x) d x\right) \\
= & I+I I+I I I+I V+V+V I .
\end{aligned}
$$

Using estimates (2.14) and (2.21), we obtain an estimate for $I I$,

$$
M^{n-1}\left|\int_{\Omega} u_{0}(x) W(x) \cdot \nabla \bar{v}_{1}(x) d x\right| \leq C\|W\|_{L^{\infty}(\Omega)} \omega\left(M^{-1}\right) .
$$


From estimate (2.16) and (2.20), we obtain an estimate for $I I I$,

$$
\begin{aligned}
M^{n-1}\left|\int_{\Omega} u_{1}(x) W(x) \cdot \nabla \bar{v}(x) d x\right| & \leq M^{n-1}\|W\|_{L^{\infty}(\Omega)}\left\|u_{1} / \delta\right\|_{L^{2}(\Omega)}\|\delta \nabla v\|_{L^{2}(\Omega)} \\
& \leq C \omega\left(M^{-1}\right)\|W\|_{L^{\infty}(\Omega)} .
\end{aligned}
$$

The term $I V$ is estimated using Cauchy-Schwarz, (2.18) and (2.22) to obtain

$$
\begin{aligned}
M^{n-1}\left|\int_{\Omega} \bar{v}_{1}(x) W(x) \cdot \nabla u_{0}(x) d x\right| & \leq C\|W\|_{L^{\infty}(\Omega)} M^{n-1}\left\|v_{1} / \delta\right\|_{L^{2}(\Omega)}\left\|\delta \nabla u_{0}\right\|_{L^{2}(\Omega)} \\
& \leq C \omega\left(M^{-1}\right)\|W\|_{L^{\infty}(\Omega)} .
\end{aligned}
$$

To estimate $V$, we use Cauchy-Schwarz, (2.15) and (2.19) to obtain

$$
\begin{aligned}
M^{n-1}\left|\int_{\Omega} \bar{v}(x) W(x) \cdot \nabla u_{1}(x) d x\right| & \leq C M^{n-1}\|W\|_{L^{\infty}(\Omega)}\|v\|_{L^{2}(\Omega)}\left\|\nabla u_{1}\right\|_{L^{2}(\Omega)} \\
& \leq C \omega\left(M^{-1}\right)\|W\|_{L^{\infty}(\Omega)} .
\end{aligned}
$$

To estimate the last term, $V I$, we use that $W$ and $q$ are bounded and estimates $(2.17)$ and (2.19) to obtain

$$
M^{n-1}\left|\int_{\Omega}(W(x) \cdot W(x)+q(x)) u(x) \bar{v}(x) d x\right| \leq C \omega\left(M^{-1}\right) .
$$

We study the main term, $I$ :

$$
\begin{aligned}
& M^{n-1} i \int_{\Omega} W(x) \cdot\left(u_{0}(x) \nabla \bar{v}_{0}(x)-\bar{v}_{0}(x) \nabla u_{0}(x)\right) d x \\
& =2 M^{n-1} N\left(\int_{\Omega} W(x) \cdot \alpha \eta_{M}(x)^{2} \exp (-2 N \rho(x)) d x\right) \\
& =2 M^{n-1} N W(0) \cdot \alpha \int_{\Omega} \exp (-2 N \rho(x)) \eta_{M}(x)^{2} d x \\
& \quad+2 M^{n-1} N \int_{\Omega} \alpha \cdot(W(x)-W(0)) \exp (-2 N \rho(x)) \eta_{M}(x)^{2} d x \\
& =W(0) \cdot \alpha+o(1)
\end{aligned}
$$

by Lemma 2.1 and the continuity of $W$.

This completes the proof of our theorem.

Proof of Corollary 1.2. We consider two magnetic Schrödinger operators which satisfy the hypotheses of Theorem 1.1 in a $C^{1}$-domain $\Omega$. Fix $x_{0}$ in $\partial \Omega$ and $\alpha$ a tangent vector to $\partial \Omega$. Subtracting the expressions for the recovery of $W_{1}$ and $W_{2}$ from Theorem 1.1, we obtain

$$
\begin{aligned}
\left|\alpha \cdot\left(W_{1}\left(x_{0}\right)-W_{2}\left(x_{0}\right)\right)\right| & =\lim _{M \rightarrow \infty}\left|\left\langle\left(\Lambda_{W_{1}, q_{1}}-\Lambda_{W_{2}, q_{2}}\right) f_{M}, f_{M}\right\rangle\right| \\
& \leq \lim _{M \rightarrow \infty}\left\|\Lambda_{W_{1}, q_{1}}-\Lambda_{W_{2}, q_{2}}\right\|\left\|f_{M}\right\|_{L^{2}(\Omega)}^{2} \\
& =\left\|\Lambda_{W_{1}, q_{1}}-\Lambda_{W_{2}, q_{2}}\right\|
\end{aligned}
$$


Thus, if $\alpha$ is unit tangent vector to $\partial \Omega$ at $x_{0}$, we obtain

$$
\left|\alpha \cdot\left(W_{1}\left(x_{0}\right)-W_{2}\left(x_{0}\right)\right)\right| \leq\left\|\Lambda_{W_{1}, q_{1}}-\Lambda_{W_{2}, q_{2}}\right\| .
$$

Choosing $x_{0}$ so that $\left|\left(W_{1}\left(x_{0}\right)-W_{2}\left(x_{0}\right)\right)_{\text {tan }}\right|$ is a maximum and $\alpha$ parallel to the real part and then the imaginary part of $w=\left(W_{1}\left(x_{0}\right)-W_{2}\left(x_{0}\right)\right)_{\tan }$ gives

$$
\left|\left(W_{1}\left(x_{0}\right)-W_{2}\left(x_{0}\right)\right)_{t a n}\right| \leq\left(|\operatorname{Re} w|^{2}+|\operatorname{Im} w|^{2}\right)^{1 / 2} \leq \sqrt{2}\left\|\Lambda_{W_{1}, q_{1}}-\Lambda_{W_{2}, q_{2}}\right\| .
$$

Proof of Theorem 1.3. We fix $x_{0} \in \partial \Omega$ and $\alpha$ a vector which is tangent to the boundary at $\partial \Omega$. We let $f_{M}(x)=M^{(n-1) / 2} u_{0}(x)$ as in (3.1) in the study of the magnetic Schrödinger operator. We have

$$
\left\langle\left(\Lambda_{W}-\Lambda_{0}\right) f_{M}, f_{M}\right\rangle=-2 M^{n-1} \int_{\Omega} \bar{v}(x) W(x) \cdot \nabla u(x) d x .
$$

If we imitate the arguments we used for the magnetic Schrödinger operator, we obtain that

$$
\left\langle\left(\Lambda_{W}-\Lambda_{0}\right) f_{M}, f_{M}\right\rangle=-2 M^{n-1} \int_{\Omega} \bar{v}_{0}(x) W(x) \cdot \nabla u_{0}(x) d x+o(1) .
$$

Recalling the definition of $u_{0},(2.2)$, we obtain

$$
\lim _{M \rightarrow \infty}-2 M^{n-1} \int_{\Omega} \bar{v}_{0}(x) W(x) \cdot \nabla u_{0}(x) d x=-\left(i \alpha+\nu\left(x_{0}\right)\right) \cdot W\left(x_{0}\right) .
$$

Since we may compute $( \pm i \alpha+\nu(x)) \cdot W\left(x_{0}\right)$ for all unit tangent vectors $\alpha$, we may recover $W\left(x_{0}\right)$.

Remark. Here, we give the changes that are needed to two dimensions.

If we assume the domain is $C^{1, \beta}$ and the dimension is two, then we have that the estimate (2.13) will hold with $\kappa=1$. (See K.O. Widman [15] for a simple argument if $n \geq 3$. His argument extends to two dimensions with minor changes.) Applying the remainder of the argument in Lemma 2.5, we conclude that

$$
\left|v_{1}(x)\right| \leq C M^{-1 / 2} N^{-1 / 2} /|x|, \quad|x|>8 M^{-1}
$$

Integrating this inequality and using that $\Omega$ is bounded gives

$$
\left\|v_{1}\right\|_{L^{2}(\Omega)} \leq C(\log (M))^{1 / 2} M^{-1 / 2} N^{-1 / 2} .
$$

Thus, we replace (2.19) and (2.20) in Lemma 2.5 by

$$
\|v\|_{L^{2}(\Omega)}+\|\delta \nabla v\|_{L^{2}(\Omega)} \leq C M^{-1 / 2} N^{-1 / 2}(\log M)^{1 / 2}
$$

In the estimates for the terms $I I I, V$, and $V I$ in the proof of Theorem 1.1, the extra factor $(\log (M))^{1 / 2}$ is multiplied by $\omega\left(M^{-1}\right)$. When $\omega(t)=C t^{\beta}$, the product $(\log (M))^{1 / 2} \omega\left(M^{-1}\right)$ will vanish in the limit. The rest of the proofs of Theorems 1.1 and 1.3 remain unchanged. 
Proof of Corollary 1.4. Fix $x_{0}$ and let $\alpha$ be a unit tangent vector to $\partial \Omega$ at $x_{0}$. From Theorem 1.3, we obtain that

$$
\left|\left(i \alpha+\nu\left(x_{0}\right)\right) \cdot\left(W_{1}\left(x_{0}\right)-W_{2}\left(x_{0}\right)\right)\right| \leq\left\|\Lambda_{W_{1}}-\Lambda_{W_{2}}\right\|
$$

Using the above inequality with $\alpha$ and $-\alpha$ and the triangle inequality, we obtain

$$
\left|\alpha \cdot\left(W_{1}\left(x_{0}\right)-W_{2}\left(x_{0}\right)\right)\right| \leq\left\|\Lambda_{W_{1}}-\Lambda_{W_{2}}\right\|
$$

and

$$
\left|\nu \cdot\left(W_{1}\left(x_{0}\right)-W_{2}\left(x_{0}\right)\right)\right| \leq\left\|\Lambda_{W_{1}}-\Lambda_{W_{2}}\right\| .
$$

Choosing a point where $W_{1}\left(x_{0}\right)-W_{2}\left(x_{0}\right)$ is maximal and then $\alpha$ parallel to the real and imaginary components of $\left(W_{1}\left(x_{0}\right)-W_{2}\left(x_{0}\right)\right)_{\text {tan }}$ and estimating the normal component of $W_{1}\left(x_{0}\right)-W_{2}\left(x_{0}\right)$ separately, gives

$$
\left.\| W_{1}\left(x_{0}\right)-W_{2}\left(x_{0}\right)\right)\left\|_{L^{\infty}(\partial \Omega)} \leq \sqrt{3}\right\| \Lambda_{W_{1}}-\Lambda_{W_{2}} \| .
$$

\section{References}

[1] G. Alessandrini. Singular solutions of elliptic equations and the determination of conductivity by boundary measurements. J. Diff. Equations, 84:252-272, 1990.

[2] R. M. Brown. Recovering the conductivity at the boundary from the Dirichlet to Neumann map: a pointwise result. J. Inverse Ill-Posed Probl., 9(6):567-574, 2001.

[3] J. Cheng, G. Nakamura, and E. Somersalo. Uniqueness of identifying the convection term. Commun. Korean Math. Soc., 16(3):405-413, 2001. Second JapanKorea Joint Seminar on Inverse Problems and Related Topics (Seoul, 2001).

[4] J. Cheng and M. Yamamoto. Determination of two convection coefficients from Dirichlet to Neumann map in the two-dimensional case. SIAM J. Math. Anal., 35(6):1371-1393 (electronic), 2004.

[5] G. Eskin and J. Ralston. Inverse scattering problem for the Schrödinger equation with magnetic potential at a fixed energy. Comm. Math. Phys., 173(1):199-224, 1995.

[6] D. Gilbarg and N.S. Trudinger. Elliptic partial differential equations of second order. Springer-Verlag, Berlin, 1983.

[7] V. Isakov. Uniqueness of recovery of some quasilinear partial differential equations. Comm. Partial Differential Equations, 26(11-12):1947-1973, 2001. 
[8] D.S. Jerison and C.E. Kenig. Boundary value problems in Lipschitz domains. In Walter Littman, editor, Studies in partial differential equations, volume 23 of MAA Studies in Mathematics, pages 1-68. Math. Assoc. Amer., Washington, D.C., 1982.

[9] G. Nakamura, Z. Sun, and G. Uhlmann. Global identifiability for an inverse problem for the Schrödinger equation in a magnetic field. Math. Ann., 303(3):377-388, 1995.

[10] M. Salo. Inverse problems for non-smooth first order perturbations of the Laplacian. Ann. Acad. Sci. Fenn. Math. Diss., 139, 2004.

[11] Ziqi Sun. An inverse boundary value problem for Schrödinger operators with vector potentials. Trans. Amer. Math. Soc., 338:953-969, 1993.

[12] A. Tamasan. On the scattering method for the $\bar{\partial}$-equation and reconstruction of convection coefficients. Inverse Problems, 20(6):1807-1817, 2004.

[13] C.F. Tolmasky. Exponentially growing solutions for nonsmooth first-order perturbations of the Laplacian. SIAM J. Math. Anal., 29(1):116-133 (electronic), 1998.

[14] C.L. Tong, J. Cheng, and M. Yamamoto. Reconstruction of convection coefficients of an elliptic equation in the plane by Dirichlet to Neumann mapping. preprint.

[15] K.0. Widman. Inequalities for the Green function and boundary continuity of the gradient of solutions of elliptic differential equations. Math. Scand., 21:17-37 (1968), 1967.

October 29, 2005 